\title{
Mathematical modeling of hydraulic resistance in pipes with rough walls
}

\begin{abstract}
In recent years, the numbers of patents have been devoted to the development of rough pipes. The technique theoretical settlement determine of factor of hydraulic resistance for round pipes with rough walls is developed on the basis of a principle of a superposition of complete viscosity in turbulent a layer mainly distinguished from the existing theories. The received results of account for the extended range of determining parameters much distinguished from appropriate given for round pipes with turbulizers, specify a level and intensification of heat exchange.
\end{abstract}

Keywords: roughness, hydro resistance, modeling, turbulent, intensification, heat transfer
Volume 2 Issue 2 - 2018

\section{Lobanov Igor Evgenjevich}

Moscow Air Institute, State Technical University, Russia

Correspondence: Lobanov Igor Evgenjevich, Moscow Air Institute, State Technical University, Volikolamskoe street 4, Moscow, Russia, Tel +7-495-489-17-58,

Email lloobbaannooff@live.ru

Received: November 14, 2017 | Published: March 23, 2018

\section{Introduction}

Study patterns of flow in pipes with rough walls can be considered relevant, as the use of rough surfaces is a method of heat transfer. In the theoretical study of heat transfer processes in heat exchangers and devices used in various fields of technology, it is very important to know the basic laws of flow in rough pipes in turbulent regime. Flow patterns for rough pipes differs from the behavior for tubes with turbulence, as indicated by both experimental ${ }^{1}$ and theoretical ${ }^{2,3}$ studies. Theoretical studies of flow in rough pipes, both experimental and theoretical, are based on the use of a logarithmic velocity profile, which to some extent simplify the mathematical model, which is especially important for large relative (relative to pipe diameter) roughness. Conditions for large roughness can be implemented, for example, in small diameter pipes. It can be compared with the conditions for pipes of small diameters with turbulence. ${ }^{4}$ Theoretical studies of pipes with rough surfaces are not numerous, sufficiently comprehensive list of studies found, for example, in, ${ }^{5-7}$ the analysis of which indicates that the theory did not come out of the logarithmic velocity profile. Generated in this study, the theory provides a more complex pattern for the coefficient of hydraulic resistance for rough pipes than existing ones, which provided him with a greater value of validity, a higher accuracy and a wider range of characteristic parameters. It should be noted that more complex patterns have occurred in the study of tubes with turbulence, ${ }^{2-4}$ which also received a more complex mathematical decision on the coefficient of hydraulic resistance, than those based on a logarithmic velocity profile. Ismagilovich et al. ${ }^{8}$ discussed the procedure to reduce the hydraulic resistance in turbulent flow comprising to introduce the anti-turbulent admixture to generate Toms effect.

\section{Mathematical modeling}

It should be noted that numerous studies rough pipes from Nikuradse studies show that at relatively high roughness turbulent flow is essentially different from the flow in smooth pipes. In US8538738, processes have been described to provide for prediction of the transition from laminar to turbulent flow on the surface. ${ }^{9}$ Rajnarayan and Sturdza ${ }^{9}$ disclosed the methods, systems and devices for estimating conversion from laminar to turbulent flow on the surface manipulating mode shape parameters.
In his stratification takes place four flow regimes: laminar (for small Reynolds numbers, regardless of the roughness that occurs in law Poiseuille) turbulent flow (for intermediate Reynolds numbers, the law of hydraulic resistance for smooth tubes) turbulent flow (for intermediate numbers Reynolds by hydraulic resistance, which is a function of the relative roughness $\bar{h}=\frac{h}{R_{0}}$ (the ratio of the average height of the roughness to the radius of the tube;

$D=2 R_{0}$ - a larger internal diameter of the pipe) and the Reynolds number) for self (at high Reynolds numbers by a factor of hydraulic resistance, which is a function only of the relative roughness). At sufficiently high relative roughness is eliminated the turbulent regime with regularity characteristic for smooth pipes. A similar situation occurs for tubes with turbulence. ${ }^{1-4} \mathrm{Liu}^{10}$ discussed the anti-drag protecting agents move the turbulent heat transfer property of the pipeline to laminar flow heat transfer property.

For tubes with a relatively low surface roughness height of the projection asymptotic behavior of the flow resistance described by the known empirical relation Nikuradse:

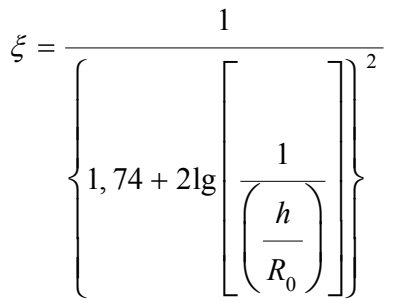

The dependence of the hydraulic resistance for rough pipes, not only on the relative roughness, and the Reynolds number $\xi=f\left(\frac{h}{R_{0}} ; \operatorname{Re}\right)$, described by the empirical formula is the best way Colebrook, which can be written as follows:

$$
\frac{1}{\sqrt{\xi}}=1,74-2 \lg \left(\frac{18,7}{\operatorname{Re} \sqrt{\xi}}+\frac{h}{R_{0}}\right)
$$

Thus, in the empirical correlations for the coefficient of hydraulic resistance of flow in rough pipes a logarithmic velocity profile. 
Hydraulic resistance coefficient for flow in straight circular tubes is determined as follows:

$$
\xi=\Delta p \frac{d}{L} \frac{2}{\tilde{n} \bar{w}_{x}^{2}}=8\left(\frac{w_{*}}{\bar{w}_{x}}\right)^{2}
$$

where $\Delta p$ - pressure drop; $d$ - diameter; $L$ - length of pipe; $\rho$ - density of coolant; $\bar{w}_{x}$ - average expenditure speed; $w_{*}$ - friction velocity.

For a straight circular pipe value speed is determined by the following integral equation:

$$
\bar{w}_{x}=2 \int_{0}^{1}(1-\bar{y}) w_{x} d y
$$

where $\bar{y}$ - relative transverse coordinate $\left(\bar{y}=\frac{y}{R_{0}}\right)$.

The integration is performed for two sites: from zero to the boundary of the viscous sublayer $\delta_{0}$ and for the core flow: $\left(\bar{\delta}=\frac{\delta_{0}}{R_{0}}\right)$

$$
\begin{aligned}
& \bar{w}_{x}=2\left[\int_{0}^{\bar{\delta}}(1-\bar{y}) w_{x} d \bar{y}+\int_{\bar{\delta}}^{1}(1-\bar{y}) w_{x} d \bar{y}\right] \\
& 1=2\left[\int_{0}^{\bar{\delta}}(1-\bar{y}) w_{x} d \bar{y}+\int_{\bar{\delta}}^{1}(1-\bar{y}) w_{x} d \bar{y}\right]
\end{aligned}
$$

Consequently, for the integration of the last expression is necessary to determine the velocity profiles $\left(\frac{w_{x}}{\bar{w}_{x}}\right)$ for each of the sub-layers. The boundary of the viscous sublayer can be determined by the following:

$$
\bar{\delta}=\delta \frac{4}{\operatorname{Re}} \sqrt{\frac{2}{\xi}}
$$

where $\delta=7,8$ - constant. ${ }^{11,12}$

The equation of motion in a straight circular pipe roughness can be written as follows:

$$
\sum_{i} v_{i} \frac{d w_{x}}{d y}=\frac{\tau_{0}}{\rho}(1-\bar{y})=w_{*}^{2}(1-\bar{y})
$$

where $\sum_{l} v_{t}$ - full kinematic viscosity; $\tau_{0}$ - shear stress at the wall.

Full kinematic viscosity based on the superposition principle is the sum of molecular viscosity; the turbulent viscosity is independent of roughness and depends on the distance from the wall, the turbulent viscosity, depending on the roughness. We must now derive formulas for the total of determining the coefficient of kinematic viscosity. The molecular kinematic viscosity is determined by the properties of the coolant and is $v$. The turbulent kinematic eddy viscosity in a circular tube, which is independent of the roughness can be postulated as a function of distance from the wall of the tube follows. Turbulent kinematic viscosity $v_{\mathrm{T} l}$ determined as the product of the mixing $l$ the characteristic speed-dynamic speed on the current radius $w_{*_{l}}$ :

$$
v_{\mathrm{T} l}=l \cdot w_{* l}
$$

The mixing 1 can be postulated as follows:

$$
l=a\left(y-\delta_{0}\right) \sqrt{1-k_{0}^{2} \bar{y}}
$$

where $k_{0}=0,97$ and $k_{0}=0,97$ - constants. ${ }^{11,12}$

Next, we have to express the dynamic speed of the current radius $w_{*}$ through dynamic speed on the tube wall $w_{*}$ :

$$
w_{* l}^{2}=w_{*}^{2}(1-\bar{y})
$$

therefore:

$$
w_{* l}=w_{*} \sqrt{1-\bar{y}}
$$

or

$$
w_{*}=\frac{w_{* l}}{\sqrt{1-\bar{y}}}
$$

Given the relationship, we obtain the final expression for the turbulent kinematic viscosity $v_{\mathrm{T} l}$, which is independent of roughness:

$\mathrm{v}_{\mathrm{T} l}=a \sqrt{1-k_{0}^{2} \bar{y}}\left(y-\delta_{0}\right) w_{*} \sqrt{1-\bar{y}}=w_{*} a\left(y-\delta_{0}\right) \sqrt{(1-\bar{y})\left(1-k_{0}^{2} \bar{y}\right)}$

The turbulent kinematic viscosity $v_{\mathrm{Th}}$, depending on the roughness is determined by the product of the characteristic length $\mathrm{L}$ the characteristic speed - dynamic speed $w_{*_{l}}$ at the current radius:

$$
v_{\mathrm{Th}}=L \cdot w_{* l}
$$

Under this theory focuses mostly pipe roughness equal height $h$. In fact, there is a certain roughness height variation. Experiments show that with small dispersion $\bar{\sigma}$ (standard deviation of the average) roughness height holds a rather abrupt transition from the smooth pipes to the regime of rough tubes, with large dispersion will be a smooth transition, since there will be a gradual withdrawal of the roughness of the viscous sublayer in the thickness of the wall layer. The characteristic length $\mathrm{L}$ for the kinematic eddy viscosity, which depends on the roughness for roughness height, is equal to:

$$
L=a\left(h-\delta_{0}\right) \sqrt{1-k_{0}^{2} \bar{y}}
$$

The final expression for the turbulent kinematic viscosity $v_{\mathrm{Th}}$, which depends on the roughness:

$$
v_{\mathrm{Th}}=w_{*} a\left(h-\delta_{0}\right) \sqrt{(1-\bar{y})\left(1-k_{0} \bar{y}\right)}
$$

Hence, the equation of motion in a straight circular pipe roughness (8) becomes (18)

$$
\left(v+w_{*} a\left(y-\delta_{0}\right) \sqrt{(1-\bar{y})\left(1-k_{0}^{2} \bar{y}\right)}+w_{*} a\left(h-\delta_{0}\right) \sqrt{(1-\bar{y})\left(1-k_{0}^{2} \bar{y}\right)}\right) \frac{d w_{x}}{d y}=w_{*}^{2}(1-\bar{y})
$$

In the future, should write (18) in the dimensionless form:

$$
\left(\frac{v}{w_{*}} \frac{1}{R_{0}}+a(\bar{y}+\bar{h}-2 \bar{\delta}) \sqrt{(1-\bar{y})\left(1-k_{0}^{2} \bar{y}\right)}\right) \frac{d\left(\frac{w_{x}}{w_{*}}\right)}{d \bar{y}}=(1-\bar{y})
$$

which for convenience should be rewritten as follows:

$$
d\left(\frac{w_{x}}{w_{*}}\right)=\frac{(1-\bar{y})}{\frac{1}{w_{*}} \frac{1}{R_{0}}+a(\bar{y}+\bar{h}-2 \bar{\delta}) \sqrt{(1-\bar{y})\left(1-k_{0}^{2} \bar{y}\right)}} d \bar{y}
$$


The desired velocity profiles $\left(\frac{w_{x}}{w_{*}}\right)$ obtained by integration of (20) provided a smooth closing of the borders sub layers under the following conditions:

$$
\begin{aligned}
& \frac{v}{w_{*}} \frac{1}{R_{0}}=\frac{4}{\operatorname{Re}} \sqrt{\frac{2}{\xi}} \\
& w_{*}=\bar{w}_{x} \sqrt{\frac{\xi}{8}}
\end{aligned}
$$

Integration is performed for the two sub-layers: 0 to the boundary of the viscous sublayer $\bar{\delta}$, for which a constantand $a \equiv 0$ from the above $\bar{\delta}$ limitsto 1 , where $a=0,39$ on the boundary condition soft closing $\frac{w_{x}}{w_{*}}=\delta$.

Hence, the equation for the coefficient of hydraulic resistance in rough tubes can be written as follows:

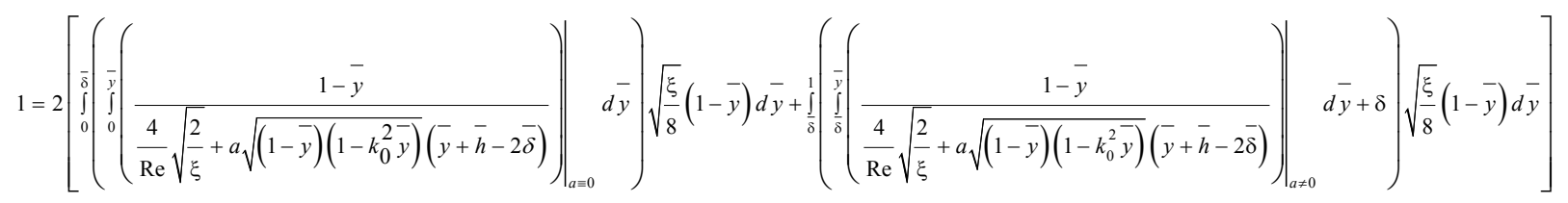

After the integration and mathematical calculations, we obtain the final equation for the transcendental of determining the coefficient of

hydraulic resistance for round rough pipes:

$$
1=\frac{\delta}{\operatorname{Re}^{3} \xi}\left(16 \delta^{3}-2 \delta \operatorname{Re}^{2} \xi+\frac{\xi \sqrt{\xi}}{2 \sqrt{2}} \operatorname{Re}^{3}\right)+\sqrt{\frac{\xi}{2}}\left(\delta \frac{4}{\operatorname{Re}} \sqrt{\frac{2}{\xi}}\right)\left(\delta \frac{4}{\operatorname{Re} \sqrt{\frac{2}{\xi}}} \int_{\frac{4}{\operatorname{Re}} \sqrt{\frac{2}{\xi}}+a \sqrt{(1-\bar{y})\left(1-k_{0}^{2} \bar{y}\right)}\left(\bar{y}+\bar{h}-\delta \frac{8}{\operatorname{Re}} \sqrt{\frac{2}{\xi}}\right)}^{-\bar{y}}(1-\bar{y}) d \bar{y}\right.
$$

The double integral in (24) cannot be expressed in a class of elementary functions. The form of the transcendental equation (24) indicates that, in calculating the hydraulic resistance in the round rough pipes is used in more complex and accurate ratio than current based (somehow) on the logarithmic velocity profile. The solution of equation (24) is best to produce numerically. As noted earlier, in this paper are modeled mostly pipe roughness with projections of equal heights. However, with the help of the model the possibility of similar modeling and when there is some deviation of the roughness height of their average value. If the variance of the mean values of roughness height $\bar{\sigma}$ is different from zero, the average height of the roughness can determine this (thus determined diffuse boundaries "roughnessviscous sublayer"):

$$
\bar{h}=\frac{\int_{\overline{\mathrm{a}}}^{\infty} p \bar{h}_{p} d \bar{h}_{p}}{\int_{\overline{\mathrm{a}}}^{\infty} p d \bar{h}_{p}}
$$

where $\bar{h}_{p}$ - values of the relative roughness height with a probability density $p$

Therefore, to calculate the average height of the roughness $\bar{h}_{p}$ , beyond the limits of the viscous sublayer $\bar{\sigma}$, need to know the probability law roughness height distribution in the pipe. For example, for a normal distribution of roughness heights transcendental equation for the pressure drop in a round of rough pipe is as follows:

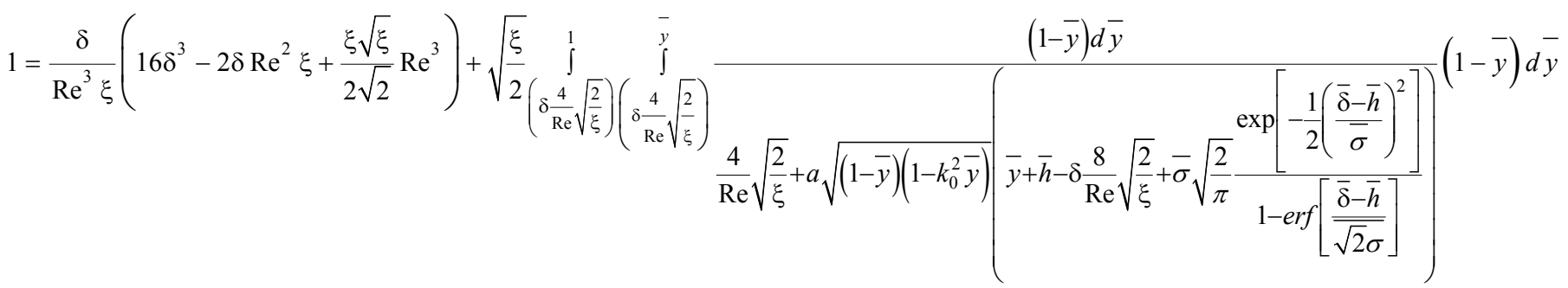

Where $\operatorname{erf}(u)=\frac{2}{\sqrt{\pi}} \int_{0}^{u} \exp \left(-u^{2}\right) d u$-Gaussian error function

In the future, should make calculations of hydraulic resistance for pipes with rough walls on received in the solution (24) for different heights of roughness and Reynolds number. This compares to bring the calculated values obtained by formula Colebrook and data for tubes with turbulators other things being equal (equal to the Reynolds number and the relative roughness height and turbulence). It should be noted that the above recent data have significant limitations on the height of vortex generator, while the equation obtained in this work, we have to great heights of roughness. Table 1 shows the calculated data for the hydraulic resistance in straight round rough tubes, obtained from the solution of the transcendental equation (24) for the 
relative heights of the turbulators $\frac{h}{R_{0}}=\frac{1}{50}=2 \cdot 10^{-2}$ and

$$
\frac{h}{R_{0}}=\frac{1}{50}=2 \cdot 10^{-2}
$$

For comparison, the similar values obtained by formula Colebrook and values for a smooth tube, obtained from the dependence Filonenko. From the data in Table 1, it is clear that the theoretical solutions are close to the formula of Colebrook for the relatively low heights of roughness. For a much wider range of heights roughness values of hydraulic resistance for round rough pipes are given in Table 2 and Table 3, in which, for comparison, the only similar relevant data, calculated by the empirical relationship Colebrook and values of hydraulic resistance for a smooth pipe from the empirical formula Filonenko. For clarity of presentation in Table 2 values of hydraulic resistance for rough pipes are given in the form of $\lg (\mathrm{Re})$ according to $\lg (100 \cdot \xi)$ for $\frac{h}{R_{0}}=\frac{1}{15} ; \frac{1}{30,6} ; \frac{1}{60} ; \frac{1}{126} ; \frac{1}{252} ; \frac{1}{507}$.

Table 3similar data are given in absolute units. As shown in Table $2 \&$ Table 3 , at relatively low altitudes of roughness at high Reynolds numbers (about $10^{6}$ ) hydraulic resistance values for rough pipes, calculated on the developed theory, approximately coincide with those values calculated by the formula Colebrook, for medium Reynolds numbers (about $10^{5}$ ) calculation formula Colebrook gives higher values.

When compared to the average roughness height theoretical values of hydraulic resistance in rough pipes are approximately equal empirical values obtained from the dependence of Colebrook, at medium and high Reynolds numbers, at low Reynolds numbers (about $10^{4}$ ) Colebrook empirical formula gives higher values. For tubes with relatively large roughness height calculated from the empirical formula Colebrook provides even inflated data on hydraulic resistance has in the entire range of Reynolds numbers. Therefore, on the basis of the data presented, we conclude that empirical correlations have a significant disadvantage compared with dependencies developed in this study, for large values of the relative roughness height $h / R_{0}$. The disadvantage of the existing theoretical and empirical relationships should be recognized that they are limited by the relative roughness height: they do not allow determine the hydraulic resistance for pipes with very high altitudes turbulence, which can be characterized, for example, rough pipes of small diameters. In the future should bring estimates of the hydraulic resistance in rough tubes, obtained by the developed dependencies, for very large values of the relative roughness height: $\mathrm{h} / \mathrm{R}_{0}=0.15 \div 0.30$. Table 4 shows the calculated values of hydraulic resistance for rough pipes are very large relative roughness height $\left(\mathrm{h} / \mathrm{R}_{0}=0.15 \div 0.30\right)$ for the typical range of Reynolds numbers $\left(\mathrm{Re}=10^{4} \div 10^{6}\right)$; for comparison, the corresponding values of hydraulic resistance for smooth pipe $\xi \mathrm{SM}\left(\mathrm{h} / \mathrm{R}_{0}=0\right)$.

Table I Hydraulic resistance in round rough pipes, calculated from the solution of the transcendental equation (24) for the relative heights of the turbulators $\mathrm{h} / \mathrm{R}_{0}=\mathrm{I} / 70$ and $\mathrm{I} / 50$.

\begin{tabular}{llllllll}
\hline \multirow{2}{*}{$\mathrm{h} / \mathrm{R}_{0} \downarrow$} & $\mathrm{Re} \rightarrow 4 \times 10^{4}$ & $6 \times 10^{4}$ & $8 \times 10^{4}$ & $10^{5}$ & $10^{6}$ & $10^{7}$ \\
\hline \multirow{2}{*}{$1 / 70=1,43 \times 10^{-2}$} & formula Filonenko & 0,02204 & 0,02008 & 0,01885 & 0,01797 & 0,01161 & 0,00812 \\
& theoretical solution & 0,03226 & 0,03328 & 0,03374 & 0,03400 & 0,03486 & 0,03494 \\
\multirow{2}{*}{$1 / 50=2,00 \times 10^{-2}$} & formula Colebrook & 0,03571 & 0,03528 & 0,03484 & 0,03466 & 0,03398 & 0,03392 \\
& theoretical solution & 0,03705 & 0,03769 & 0,03798 & 0,03816 & 0,03874 & 0,03879 \\
& formula Colebrook & 0,03935 & 0,03887 & 0,03863 & 0,03848 & 0,03794 & 0,03789 \\
\hline
\end{tabular}

Table 2 Hydraulic resistance (in the form of $\log (100 \cdot \xi)$ ) in round rough pipes, depending on the Reynolds number (in the form $\lg (\operatorname{Re})$ ), calculated from the solution of the transcendental equation (24) for a wide range of turbulence heights $h / R_{0}=1 / 15 \div 1 / 507$.

\begin{tabular}{|c|c|c|c|c|c|c|c|c|c|c|c|c|}
\hline \multirow{2}{*}{$h / R_{0}$} & \multirow{2}{*}{$\lg (100 . \xi)$} & \multicolumn{11}{|l|}{$\lg (\mathrm{Re})$} \\
\hline & & 4,0 & 4,2 & 4,4 & 4,6 & 4,8 & 5,0 & 5,2 & 5,4 & 5,6 & 5,8 & 6,0 \\
\hline \multirow{2}{*}{$1,97 \times 10^{-3}$} & theory & - & - & - & - & - & - & - & 0,242 & 0,270 & 0,284 & 0,291 \\
\hline & Colebrook & - & - & - & - & - & - & - & 0,316 & 0,308 & 0,302 & 0,298 \\
\hline \multirow{2}{*}{$3,97 \times 10^{-3}$} & theory & - & - & - & - & - & - & $0,34 I$ & 0,359 & 0,368 & 0,374 & 0,377 \\
\hline & Colebrook & - & - & - & - & - & - & 0,389 & 0,382 & 0,377 & 0,374 & 0,372 \\
\hline \multirow{2}{*}{$7,94 \times 10^{-3}$} & theory & - & - & - & - & - & 0,440 & 0,451 & 0,457 & 0,461 & 0,463 & 0,465 \\
\hline & Colebrook & - & - & - & - & - & 0,469 & 0,463 & 0,459 & 0,457 & 0,455 & 0,454 \\
\hline \multirow{2}{*}{$1,67 \times 10^{-2}$} & theory & - & - & - & 0,537 & 0,548 & 0,555 & 0,558 & 0,560 & 0,562 & 0,563 & 0,563 \\
\hline & Colebrook & - & - & - & 0,572 & 0,565 & 0,560 & 0,557 & 0,555 & 0,554 & 0,553 & 0,553 \\
\hline \multirow{2}{*}{$3,27 \times 10^{-2}$} & theory & - & 0,632 & 0,643 & 0,648 & 0,652 & 0,654 & 0,655 & 0,655 & 0,656 & 0,656 & 0,656 \\
\hline & Colebrook & - & 0,679 & 0,670 & 0,664 & 0,660 & 0,658 & 0,656 & 0,655 & 0,655 & 0,654 & 0,654 \\
\hline \multirow{2}{*}{$6,67 \times 10^{-2}$} & heory & 0,754 & 0,755 & 0,756 & 0,756 & 0,756 & 0,756 & 0,756 & 0,756 & 0,756 & 0,756 & 0,756 \\
\hline & Colebrook & 0,796 & 0,789 & 0,784 & 0,781 & 0,779 & 0,778 & 0,777 & 0,777 & 0,777 & 0,776 & 0,776 \\
\hline 0 & Filonenko & 0,497 & 0,443 & 0,392 & 0,344 & 0,298 & 0,255 & 0,213 & 0,174 & 0,136 & 0,100 & 0,065 \\
\hline
\end{tabular}


Table 3 Hydraulic resistance in round rough pipes, depending on the Reynolds number, calculated from the solution of the transcendental equation (24) for a wide range of relative heights of turbulators.

\begin{tabular}{|c|c|c|c|c|c|c|c|c|c|c|c|c|}
\hline \multirow{2}{*}{$h / R_{0}$} & \multirow{2}{*}{$\xi$} & \multicolumn{11}{|l|}{$\operatorname{Re}$} \\
\hline & & $10^{4}$ & $1,58 \times 10^{4}$ & $2,51 \times 10^{4}$ & $3,98 \times 10^{4}$ & $6,31 \times 10^{4}$ & $10^{5}$ & $1,58 \times 10^{5}$ & $2,51 \times 10^{5}$ & $3,98 \times 10^{5}$ & $6,31 \times 10^{5}$ & $10^{6}$ \\
\hline \multirow{2}{*}{${ }_{3}^{1,97 \times 10^{-}}$} & theory & - & - & - & - & - & - & - & 0,01745 & 0,01861 & 0,01921 & 0,01956 \\
\hline & Colebrook & - & - & - & - & - & - & - & 0,02072 & 0,02032 & 0,02005 & 0,01987 \\
\hline \multirow{2}{*}{$\begin{array}{l}3,97 \times 10^{-} \\
\frac{1}{-}\end{array}$} & theory & - & - & - & - & - & - & 0,02195 & 0,02285 & 0,02335 & 0,02365 & 0,02383 \\
\hline & Colebrook & - & - & - & - & - & - & 0,02448 & 0,02408 & 0,02383 & 0,02366 & 0,02355 \\
\hline \multirow{2}{*}{$\begin{array}{l}7,94 \times 10^{-} \\
3\end{array}$} & theory & - & - & - & - & - & 0,02752 & 0,02824 & 0,02866 & 0,02891 & 0,02906 & 0,02916 \\
\hline & Colebrook & - & - & - & - & - & 0,02943 & 0,02904 & 0,02879 & 0,02862 & 0,02852 & 0,02845 \\
\hline \multirow{2}{*}{${ }_{2}^{1,67 \times 10^{-}}$} & theory & - & - & - & 0,03443 & 0,03533 & 0,03585 & 0,03616 & 0,03635 & 0,03646 & 0,03653 & 0,03658 \\
\hline & Colebrook & - & - & - & 0,03730 & 0,03671 & 0,03633 & 0,03608 & 0,03592 & 0,03582 & 0,03576 & 0,03572 \\
\hline \multirow{2}{*}{$\begin{array}{l}3,27 \times 10^{-} \\
-\end{array}$} & theory & - & 0,04289 & 0,04395 & 0,0445 I & 0,04484 & 0,04503 & 0,04515 & 0,04523 & 0,04527 & 0,04530 & 0,04532 \\
\hline & Colebrook & - & 0,04770 & 0,04676 & 0,04615 & 0,04575 & 0,04549 & 0,04533 & 0,04523 & 0,04516 & 0,04512 & 0,04509 \\
\hline \multirow{2}{*}{${ }_{2}^{6,67 \times 10^{-}}$} & theory & 0,05673 & 0,05690 & 0,05697 & 0,05700 & 0,05701 & 0,05702 & 0,05702 & 0,05702 & 0,05702 & 0,05702 & 0,05702 \\
\hline & Colebrook & $0,0625 \mathrm{I}$ & 0,06150 & 0,06085 & 0,06044 & 0,06017 & $0,0600 \mathrm{I}$ & 0,05990 & 0,05983 & 0,05979 & 0,05976 & 0,05974 \\
\hline 0 & Filonenko & $0,03 \mid 44$ & 0,02774 & 0,02466 & 0,02207 & 0,01986 & 0,01797 & 0,01634 & 0,01492 & 0,01367 & 0,01258 & 0,01161 \\
\hline
\end{tabular}

Table 4 Hydraulic resistance in round rough pipes, calculated from the solution of the transcendental equation (24) for very high relative roughness heights $\left(h / R_{0}=0.15 \div 0.30\right)$, depending on the Reynolds number.

\begin{tabular}{|c|c|c|c|c|c|}
\hline \multirow{2}{*}{$h / R_{0}$} & \multicolumn{5}{|l|}{$\operatorname{Re}$} \\
\hline & $10^{4}$ & $5 \times 10^{4}$ & $10^{5}$ & $5 \times 10^{5}$ & $10^{6}$ \\
\hline 0,15 & 0,075 II & 0,07358 & 0,07338 & 0,07323 & 0,07321 \\
\hline 0,20 & 0,08177 & 0,07988 & 0,07964 & 0,07945 & 0,07943 \\
\hline 0,25 & 0,0869| & 0,08480 & 0,08454 & 0,08433 & 0,08430 \\
\hline 0,30 & 0,09104 & 0,08879 & 0,0885 I & 0,08828 & 0,08826 \\
\hline 0 & $0,03 \mid 44$ & 0,02093 & 0,01797 & $0,0|3| I \mid$ & 0,01161 \\
\hline
\end{tabular}

The data presented indicate that the pressure drop in rough tubes great height roughness increases at low Reynolds numbers from about 2.4 to 2.9 times compared to the smooth tube with increasing height roughness with $h / R_{0}=0.15$ to $h / R_{0}=0.30$, for medium Reynolds numbers, this increase is already about 4.1 and 4.9 paz respectively, for the big-about 6.3 to 7.6. When the Reynolds number increases with $10^{4}$ to $10^{6}$ hydraulic resistance of rough pipes compared to a smooth tube increases by about 2.6 times for the considered range of relative roughness height, with an increase in the relative roughness height twice (about $h / R_{0}=0.15$ to $h / R_{0}=0.30$ ) corresponding increase in the relative pressure drop of about 1.2 times. Consequently, the increase in the height of roughness and an increase in the Reynolds number is a significant increase in pressure drop in rough tubes to the smooth tube. The discrepancy between the solution for the hydraulic resistance for rough pipes, obtained in this study, with the decision based on a logarithmic velocity profile is of the order $(10 \div 15) \%$ for the relatively high roughness height at low Reynolds numbers, with a decrease in the height of roughness and an increase Reynolds discrepancy disappears.

In the future, it is necessary to conduct a comparative analysis of the estimated values of hydraulic resistance for rough pipes and tubes with turbulators other things being equal (equal heights roughness $h / R_{0}$ and turbulence, the equality of the Reynolds number Re). Table 5 the values of hydraulic resistance for round rough pipes, calculated as on the developed theory and the empirical formula Colebrook, which are compared with experimental data for pipes with periodic transverse in turbulence flow in circular pipes ${ }^{1}$ provided $h / R_{0}=i d e m$ and $\mathrm{Re}=i d e m$ $\left(h / R_{0}=0.01 \div 0,13 ; t / D=0.25 \div 1.00 ; \operatorname{Re}=10^{4} \div 2 * 10^{5}\right)$; for comparison the hydraulic resistance for a smooth tube, calculated from the empirical formula Filonenko. Comparative analysis of the hydraulic resistance in rough pipes and tubes with turbulence presented in Table 5 show that for small relative roughness height $\left(\frac{h}{R_{0}}=\frac{1}{20}\right)$ pressure drop in rough tubes at low Reynolds numbers is about the same as in the tubes with turbulators equal altitudes throughout the range considered the relative pitches between turbulence and at high Reynolds numbers-about twice as much.

At an average altitude of relative roughness $\left(\frac{h}{R_{0}}=\frac{1}{20}\right)$ pressure drop in rough tubes approximates hydraulic resistance in the pipes with turbulence: a small step between turbulence $\left(\frac{t}{D}=\frac{1}{2}\right)$ at high Reynolds numbers, the average step $\left(\frac{t}{D}=\frac{1}{2}\right)$-at medium Reynolds numbers, a large step $\left(\frac{t}{D}=1\right)$ - at low Reynolds numbers. At high altitudes, the relative roughness $\left(\frac{h}{R_{0}}=\frac{1}{10}\right)$ hydraulic resistance of rough tubes approximately the hydraulic resistance in the tubes with turbulators with a big step between turbulence at high Reynolds numbers.

Hydraulic resistance of rough tubes with large relative roughness height for the remaining cases-for small, medium and large steps between turbulence at low and medium Reynolds numbers, as well as small and medium-sized steps between turbulence at high Reynolds numbers-always much lower than for pipes with turbulence, other things being equal, $\left(h / R_{0}=i d e m\right.$ and $\left.\mathrm{Re}=i d e m\right)$. For very high 
roughness height $\left(\operatorname{Re} \geq 10^{6}\right)^{\text {hydraulic resistance of rough pipes is }}$ much lower than for tubes with turbulators other things being equal

for all the considered range of geometrical parameters of turbulence and flow regimes coolants $\left(h / R_{0}=0,11 \div 0,13 ; t / D=0.25 \div 1.00 ; \mathrm{Re}\right.$ $=10^{4} \div 2 * 10^{5}$ ). The data in Table 5 to hydraulic resistance data for rough pipes for very high Reynolds numbers $\left(\operatorname{Re} \geq 10^{6}\right)$ indicate that, for low and medium heights roughness theoretical solution obtained in this work, and the empirical formula of Colebrook give approximately equal results, and for high and very high heights roughness dependence Colebrook clearly gives higher values.Hence, it is proved that the theory has generated over the existing empirical formulas distinct advantage in the high and very high relative roughness height and very high Reynolds numbers $\left(h / R_{0}>0.10 ; \operatorname{Re} \in\left[10^{6} \div 10^{9}\right]\right)$

Table 5 Comparative analysis of hydraulic resistance values for round rough pipes calculated according to the developed theory, according to the empirical Colebrook formula and experimental data for pipes with periodic transversely located turbulators in round tubes for $h / R_{0}=0.01 \div 0.13 ; t / D=0.25 \div 1.00$; $\operatorname{Re}=10^{4} \div 4 \div 10^{5}$.

\begin{tabular}{|c|c|c|c|c|c|c|c|c|c|c|c|}
\hline \multirow{2}{*}{$h / R_{0}$} & \multirow{2}{*}{$t / D$} & \multirow{2}{*}{$\xi$} & \multicolumn{9}{|l|}{$\operatorname{Re}$} \\
\hline & & & $10^{4}$ & $2 \times 10^{4}$ & $4 \times 10^{4}$ & $10^{5}$ & $2 \times 10^{5}$ & $4 \times 10^{5}$ & $10^{6}$ & $10^{7}$ & $10^{9}$ \\
\hline \multirow{5}{*}{0,01} & 0,25 & Kalinin & 0,04588 & 0,03618 & 0,02953 & 0,02135 & 0,01795 & 0,01472 & - & - & - \\
\hline & 0,50 & Kalinin & 0,04240 & 0,02980 & 0,02595 & 0,02135 & 0,01795 & 0,01359 & - & - & - \\
\hline & 1,00 & Kalinin & 0,03322 & 0,02847 & 0,02416 & 0,01975 & 0,01601 & 0,01359 & - & - & - \\
\hline & - & theory & - & - & 0,02709 & 0,02997 & $0,0307 \mid$ & 0,03106 & $0,03 \mid 25$ & $0,03 \mid 37$ & $0,03 \mid 38$ \\
\hline & - & Colebrook & 0,03765 & 0,03448 & 0,03258 & 0,03130 & 0,03083 & 0,03059 & 0,03045 & 0,03036 & 0,03035 \\
\hline \multirow{5}{*}{0,05} & 0,25 & Kalinin & 0,10378 & 0,08780 & 0,07495 & 0,05907 & 0,05087 & 0,04403 & - & - & - \\
\hline & 0,50 & Kalinin & 0,09176 & 0,07716 & 0,06801 & 0,05427 & 0,04818 & 0,03737 & - & - & - \\
\hline & 1,00 & Kalinin & 0,04430 & 0,03884 & 0,04072 & 0,03594 & 0,03292 & 0,02453 & - & - & - \\
\hline & - & theory & 0,05039 & 0,05134 & 0,05170 & 0,05189 & 0,05194 & 0,05197 & 0,05199 & 0,05199 & 0,05200 \\
\hline & - & Colebrook & 0,05629 & $0,0547 \mid$ & 0,05389 & 0,05338 & 0,05321 & 0,05313 & 0,05308 & 0,05304 & 0,05304 \\
\hline \multirow{5}{*}{0,10} & 0,25 & Kalinin & $0,|835|$ & 0,16762 & 0,16019 & 0,14625 & 0,13166 & 0,11424 & - & - & - \\
\hline & 0,50 & Kalinin & 0,19933 & 0,19156 & 0,16287 & 0,15230 & 0,12164 & 0,10442 & - & - & - \\
\hline & 1,00 & Kalinin & $0,1430 \mid$ & $0,|277|$ & 0,12305 & 0,09964 & $0,07 \mid 22$ & 0,05133 & - & - & - \\
\hline & - & theory & 0,06580 & 0,06531 & 0,06505 & 0,06489 & 0,06484 & 0,0648 I & 0,06479 & 0,06478 & 0,06478 \\
\hline & - & Colebrook & 0,07376 & 0,07264 & 0,07207 & 0,07172 & $0,07|6|$ & $0,07 \mid 55$ & 0,07152 & $0,07 \mid 49$ & $0,07 \mid 49$ \\
\hline \multirow{3}{*}{0,11} & 0,25 & Kalinin & 0,19996 & 0,18890 & 0,18122 & 0,16369 & 0,15410 & 0,13286 & - & - & - \\
\hline & - & theory & 0,06797 & 0,06735 & 0,06703 & 0,06684 & 0,06677 & 0,06674 & 0,06671 & $0,0667 \mid$ & 0,0667 I \\
\hline & - & Colebrook & 0,07692 & 0,07586 & 0,07531 & 0,07499 & 0,07188 & 0,07482 & 0,07479 & 0,07477 & 0,07477 \\
\hline \multirow{3}{*}{0,12} & 0,50 & Kalinin & 0,26894 & 0,24557 & 0,22597 & 0,20461 & 0,15710 & 0,14594 & - & - & - \\
\hline & - & theory & 0,06996 & 0,06924 & 0,06887 & 0,06865 & 0,06857 & 0,06853 & $0,0685 I$ & 0,06850 & 0,06850 \\
\hline & - & Colebrook & 0,08002 & 0,07900 & 0,07848 & 0,07816 & 0,07806 & 0,0780 I & 0,07797 & 0,07796 & 0,07795 \\
\hline \multirow{3}{*}{0,13} & 1,00 & Kalinin & 0,31640 & 0,26473 & 0,22373 & 0,17792 & $0,142 \mid 4$ & 0,11323 & - & - & - \\
\hline & - & theory & $0,07 \mid 80$ & 0,07099 & 0,07058 & 0,07033 & 0,07025 & 0,07020 & 0,07018 & 0,07017 & 0,07016 \\
\hline & - & Colebrook & 0,08306 & 0,08207 & 0,08157 & 0,08127 & 0,08117 & $0,081 / 2$ & 0,08109 & 0,08107 & 0,08107 \\
\hline 0 & - & Filonenko & $0,03 \mid 44$ & 0,02612 & 0,02204 & 0,01797 & 0,01559 & 0,01366 & $0,01|16|$ & 0,00812 & 0,00460 \\
\hline
\end{tabular}

\section{Conclusion}

a. The technique of determining the theoretical calculation of the coefficient of hydraulic resistance for round tubes with rough walls, based on the principle of superposition full viscosity in the turbulent layer, mainly differs from existing theories.

b. Resulting in a more accurate solution for the flow resistance in rough pipes has a notable advantage over existing solutions and it should be used in the calculation, although certainly higher complexity.

c. For small and medium roughness height theoretical values of hydraulic resistance in rough tubes approximately correspond to empirical values-with a further increase of the relative roughness height mainly theoretical values differ from the 
empirical values, including, at very high Reynolds numbers .

d. Theoretical solutions obtained in this study showed that the increase in the relative values of the transactional hydraulic resistance $\frac{\xi}{\xi_{S M}}$ for tubes with very high values of the relative roughness makes a significant contribution to the increase in the height of roughness $\mathrm{h} / \mathrm{R} 0$, and an increase in the Reynolds number Re.

e. Comparative analysis of the calculated values of hydraulic resistance in rough tubes with similar experimental values for tubes with periodic transverse in turbulence flow showed that the rough is very large relative roughness height hydraulic resistance is always lower than for tubes with turbulators other things being equal, a small, medium and large heights found the limits of their approximate match other things being equal: the smaller the Reynolds number Re, the greater should be the relative spacing between the turbulence $\mathrm{h} / \mathrm{R} 0$.

f. The main advantage of the solutions obtained by the theory developed in comparison with empirical dependencies is that they allow you to calculate the pressure drop in rough tubes for large and very large relative roughness height including for large Reynolds numbers, which is typical, for example, for small diameter pipes.

g. Result of the calculation of hydraulic resistance for round rough pipes for an extended range of characteristic parameters that are significantly different from the corresponding data for round tubes with turbulence, indirectly indicate the level of heat transfer through the use of rough tubes instead of smooth.

\section{Acknowledgement}

None.

\section{Conflict of interest}

The author confirms that this article content has no conflict of interest.

\section{References}

1. Kalinin EK, Dreytzer GA, Kopp IZ et al. Effective surfaces of heat exchange. Energoatomizdat: Moscow; 1998. 408p.

2. Lobanov IE. Heat transfer in turbulent flow in a plane channel with evenly spaced surface unilateral-lateral turbulence flow. Bulletin of Mechanical Engineering. 2012; 8:13-17.

3. Lobanov IE. Mathematical modeling of intensified-ion heat transfer in turbulent flow in pipes with turbulization-congestion based on the equation of balance of turbulent fluctuating energy. Sectoral Aspects of Engineering. 2012;12:24-34.

4. Lobanov IE. Modeling the Structure of Vortex Zones between Periodic Superficially Located Energizers of the Flow of a Rectangular Cross Section. Mathematical Models and Computer Simulations. 2013;5(1):6374

5. Parakram P, James H, Manhar D, et al. Numerical modeling of turbulence and its effect on ocean current turbines. International Journal of Marine Energy. 2017;17:84-97.

6. Ljahov VK. Method of relative conformity at accounts turbulent parietal of flows. Publishing house of the Saratov University: Russia; 1975. 123p.

7. Ljahov VK, Migalin VK. Effect thermal, or diffusive, roughness. Publishing house of the Saratov University: Russia; 1989. 176p.

8. Ismagilovich RS, Janovich GAG, Mukhutdinovich GM, et al. Method of reducing hydraulic resistance in turbulent flow. EMT Research AS: USA; 2006.

9. Rajnarayan Dev, Sturdza P. Predicting transition from laminar to turbulent flow over a surface using mode-shape parameters. Aerion Technologies Corp: USA; 2013.

10. Liu L. Oil and gas pipeline drag reducing and heat preservation method. Xi'an Jiaotong University: China; 2012.

11. Milliontshikov MD. Turbulent of current in a boundary layer and in pipes. Nuclear Energy. 1970;28(3):207-214.

12. Milliontshikov MD. Turbulent is warm and mass exchange in pipes with smooth and rough walls. Nuclear Energy. 1971;31(3):199-204. 